\title{
Modeling the Sensing Element of Pressure Nanosensors as Simply Supported Size-Dependent Rectangular Plate ${ }^{\dagger}$
}

\author{
Marina Barulina and Dmitry Kondratov \\ Institute of Precision Mechanics and Control, Russian Academy of Sciences, Saratov, Russia \\ + Presented at the Entropy 2021: The Scientific Tool of the 21st Century, 5-7 May 2021; Available online: \\ https://sciforum.net/conference/Entropy2021/.
}

Published: 5 May 2021

The study of the dynamics of the nanoelectromechanical sensor (NEMS) is currently relevant since they are the next step in the evolution and miniaturization of sensors. Due to the nanosized of sense elements and other components of NEMS, they need in non-classical approaches for the study of their dynamics. Furthermore, the development of these non-classical approaches is a fundamental problem, and many scientists are working on its resolving. One more significant problem is the application of these non-classical approaches to components of the different kinds of NEMS and the obtainment the mathematical models are ready to use for a practical purpose.

In the paper, the mathematical model of the sensing element of pressure nanosensors was constructed based on the new modified couple-stress theory and the third-order plate theory. The sensing element was considered as a simply supported rectangular nanoplate under the distributed force at the bottom of the plate. The dynamic version of the principle of virtual displacements was used for obtaining the differential equations of motion and natural boundary conditions.

A series of computational experiments were carried out for an orthotropic nanoplate. Some combinations of parameters of the mathematical model are found for which chaotic motion is possible. 